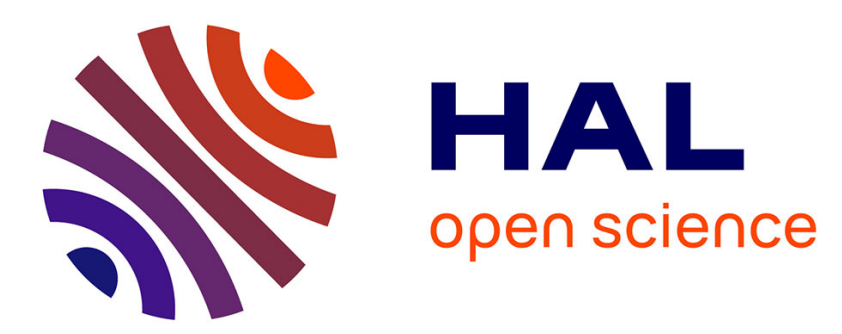

\title{
EEG sensor selection by sparse spatial filtering in P300 speller brain-computer interface
}

Bertrand Rivet, Hubert Cecotti, Ronald Phlypo, Olivier Bertrand, Emmanuel Maby, Jérémie Mattout

\section{- To cite this version:}

Bertrand Rivet, Hubert Cecotti, Ronald Phlypo, Olivier Bertrand, Emmanuel Maby, et al.. EEG sensor selection by sparse spatial filtering in P300 speller brain-computer interface. EMBC 2010 32nd Annual International Conference of the IEEE Engineering in Medicine and Biology Society, Aug 2010, Buenos Aires, Argentina. pp.n.c. hal-00517388

\section{HAL Id: hal-00517388 \\ https://hal.science/hal-00517388}

Submitted on 14 Sep 2010

HAL is a multi-disciplinary open access archive for the deposit and dissemination of scientific research documents, whether they are published or not. The documents may come from teaching and research institutions in France or abroad, or from public or private research centers.
L'archive ouverte pluridisciplinaire HAL, est destinée au dépôt et à la diffusion de documents scientifiques de niveau recherche, publiés ou non, émanant des établissements d'enseignement et de recherche français ou étrangers, des laboratoires publics ou privés. 


\title{
EEG sensor selection by sparse spatial filtering in P300 speller brain-computer interface
}

\author{
Bertrand Rivet, Hubert Cecotti, Ronald Phlypo, Olivier Bertrand, Emmanuel Maby and Jérémie Mattout
}

\begin{abstract}
A Brain-Computer Interface (BCI) is a specific type of human-machine interface that enables communication between a subject/patient and a computer by direct control from decoding of brain activity. This paper deals with the P300speller application that enables to write a text based on the oddball paradigm. To improve the ergonomics and minimize the cost of such a BCI, reducing the number of electrodes is mandatory. We propose a new algorithm to select a relevant subset of electrodes by estimating sparse spatial filters. A $l_{1}$ norm penalization term, as an approximation of the $l_{0}$-norm, is introduced in the xDAWN algorithm, which maximizes the signal to signal-plus-noise ratio. Experimental results on 20 subjects show that the proposed method is efficient to select the most relevant sensors: from 32 down to 10 sensors, the loss in classification accuracy is less than $5 \%$.
\end{abstract}

\section{INTRODUCTION}

Brain-Computer Interfaces (BCI) are new kinds of devices, which enable direct communication between the user's brain and a computer [1], [2]: such systems allow people to communicate through direct measurements of brain activity, without requiring any movement. BCIs usually exploit cerebral activities measured by electroencephalography (EEG) and could thus be useful to patients who suffer from severe neuromuscular disorders (e.g. "locked in" patients). Current BCIs determine the user's intent from different electrophysiological signals: for instance the user may actively control some brain waves (e.g., mu or beta rhythms) or the BCI may exploit natural passive response of the brain to external stimuli (e.g., event related potentials) [1].

The BCI problem addressed in this paper concerns the P300-speller [3], [4] which enables people to write a text on a computer. It is based on the oddball paradigm: the task is to discriminate between epochs containing a P300 potential evoked by a (rare) target stimulus and epochs associated with the (frequent) non-target stimuli. To gather enough evidence and yield robust classification, each symbol/stimulus is presented/flashed several times. However, this is done at the expense of reducing the speed of the system: e.g., with 15 repetitions, about two symbols can be spelt per minute only [3]. Moreover, the signal-to-noise ratio (SNR) of EEG signals is very low, and signals often contain muscular and/or ocular artifacts. To increase SNR and hence classification accuracy, several methods based on spatial filtering have been

This work was partially supported by ANR-Tecsan RoBIK, ANR-Blanc GAZE\&EEG and ANR-DEFIS CoAdpat.

Bertrand Rivet, Hubert Cecotti and Ronald Phlypo are with GIPSAlab, CNRS-UMR 5216, Grenoble Institute of Technology, France bertrand.rivetegipsa-lab.grenoble-inp.fr

Olivier Bertrand, Emmanuel Maby and Jérémie Mattout are with INSERM U821, Institut Federatif des Neurosciences, Lyon, France proposed such as those based on independent component analysis (ICA) [5], [6] or, more recently, the xDAWN algorithm [7], [8]. These studies have shown that enhancing the P300 response using spatial filters enables to reduce the size of the required training set in order to reach a certain level of classification accuracy in the test phase. As a consequence, the ergonomics is improved as the training phase becomes shorter. Yet to further improve the BCI ergonomics, a crucial need is to reduce the number of electrodes. Indeed, using a large number of electrodes is undesirable since it increases the discomfort of the patient, the time needed to install the whole system as well as its global cost. However, only a few studies have focused on sensor selection so far. Some studies did compared a priori selected subsets in terms of subsequent classification performance (e.g., [9], [10]) while others have applied by blind approaches to select the most relevant sensors (e.g., [11]). The major drawback of the latter is its need for $\mathrm{K}$-fold cross validation, which requires a large number of training symbols. In [12], we proposed a faster and efficient sensor selection approach based on backward elimination. In the current study, the xDAWN algorithm is modified by supplementing the SNR criterion with a $l_{1}$ norm penalization term which leads to estimate sparse spatial filters, that is with only a few non-zero coefficients.

This paper is organized as follows. Section II describes the P300 subspace estimation and the associated classification issue. Section III presents the obtained results on empirical data from twenty subjects. Finally Section IV concludes the paper with comments and perspectives for future work.

\section{METHODOLOGY}

\section{A. Data description and preprocessing}

The P300-speller enables the user to select symbols (letters, digits, images). The target plays the role of an oddball and thus elicits a P300 response each time it is emphasized on screen. In our setting, a $6 \times 6$ matrix that contains all the available symbols is displayed on a computer screen (Fig. 1). To spell one symbol, the user has to focus on the one $\mathrm{s} / \mathrm{he}$ wants to spell. When the user focuses his attention on a cell of the matrix, it is possible to detect a P300 (a positive deflection in voltage at a latency of about $300 \mathrm{~ms}$ in the EEG) after the cell was intensified. Symbols are not intensified or flashed individually but together with the symbols that belong to the same matrix row or column. Row and column intensification is randomized by blocks of 12 ( 6 rows and 6 columns). The sets of 12 intensifications is repeated $N_{\text {epoch }}$ times for each symbol. Therefore, $2 N_{\text {epoch }}$ P300 responses might be detected for to identify the target recognition: each 

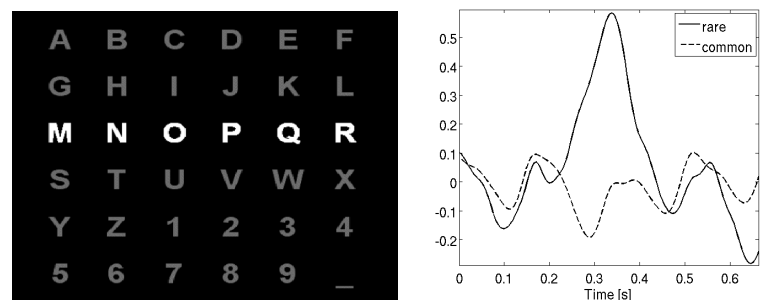

(a) P300-Speller Graphical User (b) Typical responses to tarInterface get/rare (P300) and non-target/ frequent stimuli

Fig. 1. P300-Speller BCI application. Fig. 1(a): screen display, Fig. 1(b): average responses on sensor location $\mathrm{Cz}$

symbol is uniquely defined by the intersection of one row and one column.

Data were recorded from 20 healthy subjects [13]. Two sessions were considered: one for training the classifier, the other for testing. For the training and test sessions, the subject had to write 50 and 60 predetermined symbols respectively. Each row and column in the spelling matrix was randomly intensified for $100 \mathrm{~ms}$ and the delay between two consecutive intensifications was $80 \mathrm{~ms}$, leading to an interstimulus interval (ISI) of $180 \mathrm{~ms}$. For each symbol there was 10 consecutive epochs $\left(N_{\text {epoch }}=10\right)$.

The EEG signals were initially sampled at $100 \mathrm{~Hz}$. Data preprocessing included a fourth order bandpass filter between $1 \mathrm{~Hz}$ and $20 \mathrm{~Hz}$. Finally, each sensor signal was centered and reduced so that they had a zero mean value and a standard deviation equal to one.

\section{B. xDAWN algorithm to enhance P300 potentials}

In this section, the principle of the XDAWN algorithm is briefly reprised [7], [8]. Its goal is to enhance the signal to signal-plus-noie (SSNR). This method relies on two assumptions: i) signal is made of two typical responses, one evoked by the targets and one evoked by all stimuli (target and non-target ones); ii) responses evoked by the target stimuli could be enhanced by spatial filtering. Let $X \in \mathbb{R}^{N_{t} \times N_{s}}$ denote the recorded signals, where $N_{t}$ and $N_{s}$ are the number of samples and sensors, respectively. We denote the responses synchronized with the target and non-target stimuli by $A_{1} \in \mathbb{R}^{N_{1} \times N_{s}}$ and $A_{2} \in \mathbb{R}^{N_{2} \times N_{s}}$, respectively. Thus,

$$
X=D_{1} A_{1}+D_{2} A_{2}+N,
$$

where $D_{1} \in \mathbb{R}^{N_{t} \times N_{1}}$ and $D_{2} \in \mathbb{R}^{N_{t} \times N_{2}}$ are Toeplitz matrices whose first column entries are set to zero except for those that correspond to target stimuli and all stimuli, respectively. $N_{1}$ and $N_{2}$ indicate the number of samples in responses $A_{1}$ and $A_{2}$, respectively. $N \in \mathbb{R}^{N_{t} \times N_{s}}$ denotes the residual noise.

The aim of the xDAWN algorithm is to estimate $N_{f}$ spatial filters $U_{1} \in \mathbb{R}^{N_{s} \times N_{f}}$ so that the SSNR defined by

$$
g(U)=\frac{\operatorname{Tr}\left(U^{T} \hat{\Sigma}_{1} U\right)}{\operatorname{Tr}\left(U^{T} \hat{\Sigma}_{X} U\right)}
$$

is maximum

$$
\hat{U}_{1}=\arg \max _{U_{1}}, g\left(U_{1}\right)
$$

where $\operatorname{Tr}(\cdot)$ is the trace operator and $\hat{\Sigma}_{1}=\hat{A}_{1}^{T} D_{1}^{T} D_{1} \hat{A}_{1}$, $\hat{\Sigma}_{X}=X^{T} X$. Note that $\hat{A}_{1}$ is the least mean square estimation of the unknown target evoked response $A_{1}$. Since $D_{1} A_{1}$ and $D_{2} A_{2}$ could overlap, $\hat{A}_{1}$ is estimated from

$$
\left(\begin{array}{l}
\hat{A}_{1} \\
\hat{A}_{2}
\end{array}\right)=\left(D^{T} D\right)^{-1} D^{T} X
$$

with $D=\left[D_{1}, D_{2}\right]$. To estimate the spatial filters $\hat{U}_{1}$, one computes the $\mathrm{QR}$ decompositions $X=Q_{X} R_{X}$ and $D_{1}=Q_{1} R_{1}$ [14]. Spatial filters then obtains from $\hat{U}_{1}=$ $R_{X}^{-1} \Psi_{1: N_{f}}$, where $\Psi_{1: N_{f}}$ is the concatenation of the $N_{f}$ singular vectors $\Psi_{i}$ associated with the $N_{f}$ maximum singular values $\lambda_{i}$ provided by the singular value decomposition of $R_{1} B_{1}^{T} Q_{X}=\Phi \Lambda \Psi^{T} . \Phi$ and $\Psi$ are unitary matrices, $\Lambda$ is a diagonal matrix [14] and $\hat{A}_{1}=B_{1}^{T} X$. Finally, the enhanced signals are given by

$$
\hat{S}_{1} \triangleq X \hat{U}_{1}=D_{1} A_{1} \hat{U}_{1}+D_{2} A_{2} \hat{U}_{1}+N \hat{U}_{1} .
$$

\section{Sparse estimation of spatial filters to select sensors}

A substantial drawback of the xDAWN algorithm (3) is that it provides a non-sparse spatial filters $\hat{U}_{1}$, leading thus to consider that all the sensors are necessary to achieve a suitable enhancement of response $A_{1}$ evoked by the target. However we have already shown that based on this approach, the number of sensors could still be reduced (e.g., from 32 down to 8 sensors) while keeping a high classification accuracy ( $85 \%$ instead of 92\%) [12]. Nevertheless, in this study, we describe and evaluate a more generic and simpler method to select relevant sensors. It consists of introducing a $l_{1}$-norm penalization term in the xDAWN criterion (3) as follows:

$$
\hat{\mathbf{v}}_{1}=\arg \max _{\left\|\mathbf{v}_{1}\right\|_{2}=1} f\left(\mathbf{v}_{1}, \lambda\right)
$$

with $f\left(\mathbf{v}_{1}, \lambda\right)$ defined by

$$
f\left(\mathbf{v}_{1}, \lambda\right)=g\left(\mathbf{v}_{1}\right)-\lambda \frac{\left\|\mathbf{v}_{1}\right\|_{1}}{\left\|\mathbf{v}_{1}\right\|_{2}},
$$

where hyperparameter $\lambda \geq 0$ is a penalization multiplier adjusted to control the sparsity of the spatial filter $\mathbf{v}_{1}$. Indeed, the $l_{1}$-norm penalization is well known to be a good candidate to solve sparse problem as it could be seen as an approximation of the $l_{0}$-norm which is the number of nonzero elements of a vector [15], [16]. Note that the constraint $\left\|\mathbf{v}_{1}\right\|_{2}=1$ is required since the Rayleigh quotient (first term in criterion (7)) is invariant by a scaling factor. To maximize (6), a gradient method is used:

$$
\mathbf{v}_{1}^{(k+1)}=\frac{\mathbf{v}_{1}^{(k)}+\rho^{(k)} \partial_{\mathbf{v}_{1}} f\left(\mathbf{v}_{1}^{(k)}, \lambda\right)}{\left\|\mathbf{v}_{1}^{(k)}+\rho^{(k)} \partial_{\mathbf{v}_{1}} f\left(\mathbf{v}_{1}^{(k)}, \lambda\right)\right\|_{2}},
$$

where $\partial_{\mathbf{v}_{1}} f\left(\mathbf{v}_{1}^{(k)}, \lambda\right)$ is a subgradient of $f\left(\mathbf{v}_{1}, \lambda\right)$ defined by

$$
\partial_{\mathbf{v}_{1}} f\left(\mathbf{v}_{1}^{(k)}, \lambda\right)=\frac{\partial}{\partial \mathbf{v}_{1}}\left(\frac{\mathbf{v}_{1}^{T} \hat{\Sigma}_{1} \mathbf{v}_{1}}{\mathbf{v}_{1}^{T} \hat{\Sigma}_{X} \mathbf{v}_{1}}\right)-\lambda \partial_{\mathbf{v}_{1}}\left(\frac{\left\|\mathbf{v}_{1}\right\|_{1}}{\left\|\mathbf{v}_{1}\right\|_{2}}\right),
$$


with

$$
\frac{\partial}{\partial \mathbf{v}_{1}}\left(\frac{\mathbf{v}_{1}^{T} \hat{\Sigma}_{1} \mathbf{v}_{1}}{\mathbf{v}_{1}^{T} \hat{\Sigma}_{X} \mathbf{v}_{1}}\right)=\frac{2}{\mathbf{v}_{1}^{T} \hat{\Sigma}_{X} \mathbf{v}_{1}}\left(\hat{\Sigma}_{1} \mathbf{v}_{1}-\frac{\mathbf{v}_{1}^{T} \hat{\Sigma}_{1} \mathbf{v}_{1}}{\mathbf{v}_{1}^{T} \hat{\Sigma}_{X} \mathbf{v}_{1}} \hat{\Sigma}_{X} \mathbf{v}_{1}\right)
$$

and

$$
\partial_{\mathbf{v}_{1}}\left(\frac{\left\|\mathbf{v}_{1}\right\|_{1}}{\left\|\mathbf{v}_{1}\right\|_{2}}\right)=\frac{S\left(\mathbf{v}_{1}\right) 1_{N_{s}}}{\left\|\mathbf{v}_{1}\right\|_{2}}-\frac{\left\|\mathbf{v}_{1}\right\|_{1}}{\left(\mathbf{v}_{1}^{T} \mathbf{v}_{1}\right)^{3 / 2}} \mathbf{v}_{1}
$$

where $1_{N_{s}} \in \mathbb{R}^{N_{s}}$ is a vector of ones and $S\left(\mathbf{v}_{1}\right)$ is a diagonal matrix such that

$$
S\left(\mathbf{v}_{1}\right)_{i i}= \begin{cases}1, & \text { if } \mathbf{v}_{1}(i)>0 \\ 0, & \text { if } \mathbf{v}_{1}(i)=0 \\ -1, & \text { if } \mathbf{v}_{1}(i)<0 .\end{cases}
$$

Note that i) this is only a subgradient since the $l_{1}$-norm is undifferentiable; ii) the division of $\left\|\mathbf{v}_{1}\right\|_{1}$ by the $l_{2}$-norm $\left\|\mathbf{v}_{1}\right\|_{2}$ in the penalization term in criterion (7) is to ensure that iteration $\mathbf{v}_{1}^{(k)}+\rho^{(k)} \partial_{\mathbf{v}_{1}} f\left(\mathbf{v}_{1}^{(k)}, \lambda\right)$ has a $l_{2}$-norm as close as possible to one before projection onto the $l_{2}$-ball of unit radius in order to speed up the convergence of the gradient algorithm. In this study, parameter $\lambda$ that controls the sparsity of the estimated spatial filter $\hat{\mathbf{v}}_{1}$ is chosen manually based on the number $N_{s s}$ of selected sensors. Finally, the $N_{s s}$ selected sensors corresponding to the non-zero entries in $\hat{\mathbf{v}}_{1}(6)$. The final sparse spatial filters $\hat{\mathbf{v}}_{i}^{*}$ are then computed by applying the classical (unconstrainted) xDAWN algorithm (3) to those selected sensors only.

\section{Classification}

Among the proposed classifiers that are considered for BCIs, Bayesian linear discriminant analysis (BLDA) [9] is chosen since it has proved efficient and is fully automated (i.e. no need to adjust some hyperparameter) [9]. BLDA consists of finding a discriminant vector $\mathbf{w}$ such that $\mathbf{w}^{T} \mathbf{p}$ is as close as possible to the class index $t$ associated with the corresponding feature vector $\mathbf{p}$ obtained from the concatenation of time-course samples of enhanced signals. The discriminant vector $\mathbf{w}$ is estimated from the set of pairs $\left\{\mathbf{p}_{j}, t_{j}\right\}_{1 \leq j \leq 12 N_{c} N_{\text {epoch }}}$ obtained from the $N_{c}$ symbols of the training database.

\section{RESULTS}

In this section we present the results obtained from the above described experiment using the proposed new approach for sparse spatial filtering, in comparison with classical filtering using the xDAWN algorithm. We considered epochs of $1 \mathrm{~s}$ duration for the target response $A_{1}$ and $.180 \mathrm{~s}$ for non-target response $A_{2}$. Moreover, in this preliminary study, the penalization parameter $\lambda$ was optimized manually and independently for each subject.

As $\lambda$ increases, the sparsity of the estimated spatial filters $\hat{\mathbf{v}}_{1}$ (6) grows (Fig. 2). This penalization parameter must thus be chosen carefully with respect to the desired number of sensors to be kept. On figure 2, we see the interesting evolution of the SSNR as a function of hyperparameter $\lambda$. The sparse SSNR corresponds to $g\left(\hat{\mathbf{v}}_{1}\right)$, where $\hat{\mathbf{v}}_{1}$ are the

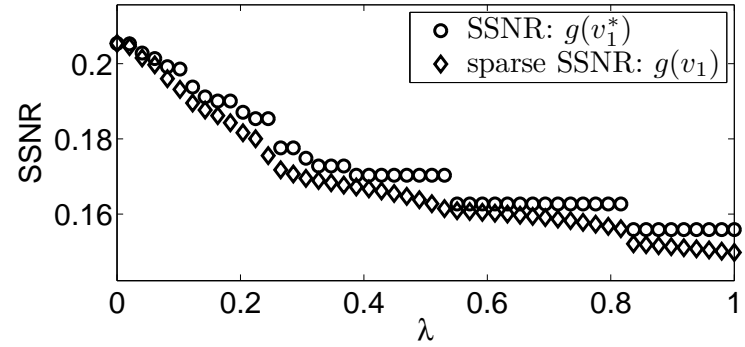

Fig. 2. Influence of penalization parameter $\lambda$ on the SSNR.

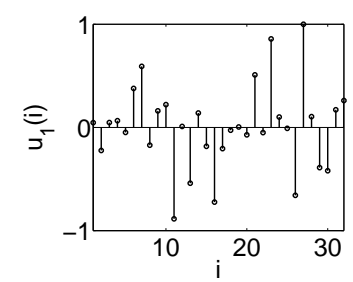

(a) Non-sparse spatial filter

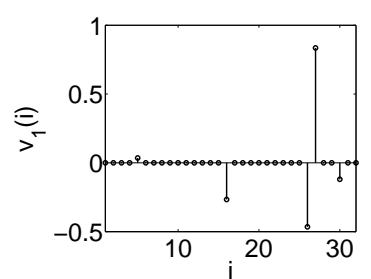

(b) Sparse spatial filter
Fig. 3. Illustration of the sparse spatial filtering: Fig. 3(a) classical xDAWN, Fig. 3(b) sparse xDAWN $(\lambda=0.4)$.

sparse spatial filters computed using the penalized criterion. The classical SSNR refers to $g\left(\hat{\mathbf{v}}_{1}^{*}\right)$, where $\hat{\mathbf{v}}_{1}^{*}$ are the spatial filters computed using the unconstrained criterion, once the sensors have been selected. We observe that the sparse SSNR follows a continuous decrease, while the classical SSNR follows a step-wise decrease where each step corresponds to a drop of one or several sensors. For the latter, the more sensors have been dropped simultaneously, the larger the drop in SSNR. This clear relationship suggests that one could fairly easily adjust the penalization parameter given a desired final number of sensors.

Fig. 3 shows an example of spatial filters estimated by classical xDAWN (Fig. 3(a)) and by sparse xDAWN (Fig. 3(b)), respectively. With $\lambda=0.4$, only 5 out of 32 sensors ends up with non-zero entries. Importantly, it appears that the remaining sensors do not necessarily correspond to those with the largest entries in $\hat{\mathbf{u}}_{1}$. Hence simple projection onto the $l_{1}$-ball or soft/hard-thresholding would not have resulted in the same optimum set of sensors.

Figure 4 depicts the classification accuracy as a function of the number of selected sensors $\left(N_{s s}\right)$. As expected, the more sensors, the higher the accuracy. However, from 10 to 32 sensors the gain in average classification accuracy is smaller than 5\%. The same effect obtains when only considering 5 epochs per symbol. Finally, we observed that for about $75 \%$ of the subjects, reducing the number of electrodes down to 8 has not a profound impact on classification accuracy (Fig. 4(b)). This is highly promising in the aim of improving the ergonomics of such BCIs.

Although the selected electrodes are specific to each subject, preliminary comparisons between subjects show that 


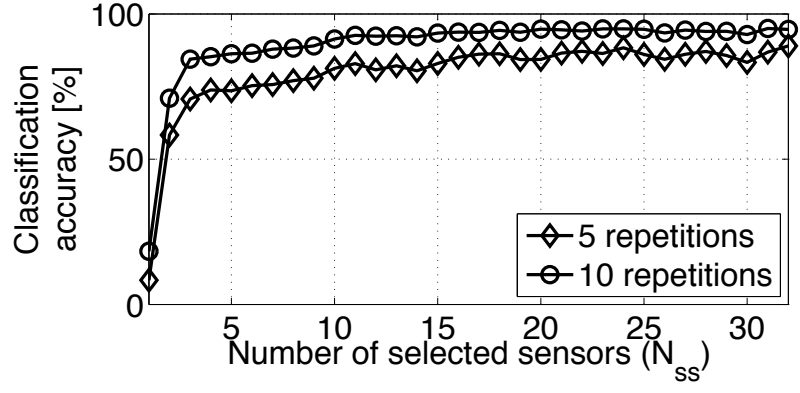

(a) Average performance

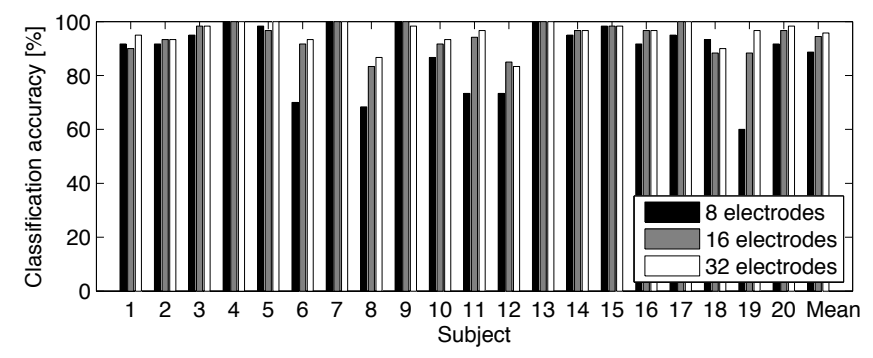

(b) Individual performance (10 repetitions)

Fig. 4. Average classification accuracy of the P300 speller versus the number of selected sensors.

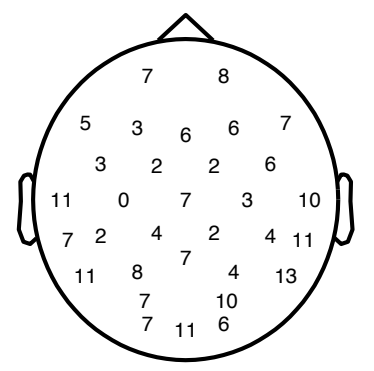

Fig. 5. Selected sensors: each number corresponds to the total times this sensor is part of the 10 most relevant sensors for a specific subject.

several sensors are common to a majority of subjects. For instance, with 10 selected sensors, five of them appear to be common to more than 10 subjects (Fig. 5). These electrodes are mainly located over occipital areas, confirming previous findings that suggested those sites sites were relevant [17], [18].

\section{CONCLUSION AND PERSPECTIVES}

In this preliminary study, a new and promising sensor selection procedure was introduced. It is based on a global criterion defined by the maximization of the signal to signal-plus-noise ratio (SSNR) penalized by a $l_{1}$-norm term to favour sparse estimates. The selected sensors are then spatially filtered by the unconstrained xDAWN algorithm leading to the largest SSNR. We established that the choice of a penalization parameter value allows to select a specific reduced subset of relevant electrodes. Namely, reducing the number of sensors by $66 \%$ yields only a slight decrease in classification accuracy (by less than 5\%). Importantly, this new method entails a global optimization approach compared to backward elimination as introduced in [12].
Further works will include a deeper analysis of the influence of the penalization parameter $\lambda$ on the criterion to propose a fully automated scheme. Then we will be able to focus on the identification of universal sensor subsets that could ensure high accuracy for every subject.

\section{REFERENCES}

[1] J. R. Wolpaw, N. Birbaumer, D. J. McFarland, G. Pfurtscheller, and T. M. Vaughan, "Brain-computer interfaces for communication and control." Clinical Neurophysiology, vol. 113, no. 6, pp. 767-791, June 2002.

[2] L. R. Hochberg, M. D. Serruya, G. M. Friehs, J. A. Mukand, M. Saleh, A. H. Caplan, A. Branner, D. Chen, R. D. Penn, and J. P. Donoghue, "Neuronal ensemble control of prosthetic devices by a human with tetraplegia," Nature, vol. 442, no. 7099, pp. 164-171, Jul. 2006.

[3] L. A. Farwell and E. Donchin, "Talking off the top of your head: toward a mental prosthesis utilizing event-related brain potentials," Electroencephalography and Clinical Neurophysiology, vol. 70, no. 6, pp. 510-523, December 1988.

[4] E. Donchin, K. Spencer, and R. Wijesinghe, "The mental prosthesis: assessing the speed of a P300-based brain-computer interface," IEEE Transactions on Rehabilitation Engineering, vol. 8, no. 2, pp. 174179 , June 2000.

[5] N. Xu, X. Gao, B. Hong, X. Miao, S. Gao, and F. Yang, "BCI Competition 2003-Data Set IIb: Enhancing P300 Wave Detection Using ICA-Based Subspace Projections for BCI Applications," IEEE Transactions on Biomedical Engineering, vol. 51, no. 6, pp. 10671072, June 2004.

[6] H. Serby, E. Yom-Tov, and G. Inbar, "An improved P300-based brain-computer interface," IEEE Transactions on Neural Systems and Rehabilitation Engineering, vol. 13, no. 1, pp. 89-98, March 2005.

[7] B. Rivet, A. Souloumiac, G. Gibert, and V. Attina, "'P300 speller" Brain-Computer Interface: Enhancement of P300 evoked potential by spatial filters," in Proc. European Signal Processing Conference (EUSIPCO), Lausanne, Switzerland, August 2008.

[8] B. Rivet, A. Souloumiac, V. Attina, and G. Gibert, "xDAWN algorithm to enhance evoked potentials: Application to brain-computer interface," IEEE Transactions on Biomedical Engineering, vol. 56, no. 8, pp. 2035-2043, August 2009.

[9] U. Hoffmann, J.-M. Vesin, T. Ebrahimi, and K. Diserens, "An efficient P300-based brain-computer interface for disabled subjects," Journal of Neuroscience Methods, vol. 167, no. 1, pp. 115-125, Jan. 2008.

[10] P. Meinicke, M. Kaper, F. Hoppe, M. Heumann, and H. Ritter, Advances in Neural Information Processing Systems 15,. MIT Press, Cambridge, MA, 2003, ch. Improving Transfer Rates in Brain Computer Interfacing: A Case Study, pp. 1107-1114.

[11] A. Rakotomamonjy and V. Guigue, "BCI Competition III: Dataset II- Ensemble of SVMs for BCI P300 Speller," IEEE Transactions on Biomedical Engineering, vol. 55, no. 3, pp. 1147-1154, March 2008.

[12] B. Rivet, A. Souloumiac, G. Gibert, V. Attina, and O. Bertrand, "Sensor selection for P300 speller brain computer interface," in Proc. European Symposium on Artificial Neural Networks, Bruges, Belgiunm, 2009, pp. 431-438.

[13] E. Maby, G. Gibert, P.-E. Aguera, M. Perrin, O. Bertrand, and J. Mattout, "The openvibe p300-speller scenario: a thorough online evaluation," in Human Brain Mapping Conference, 2010, (accepted).

[14] G. H. Golub and C. F. Van Loan, Matrix Computation, 3rd ed. Johns Hopkins University Press, 1996.

[15] P. O. Hoyer, "Non-negative matrix factorization with sparseness constraints," Journal of Machine Learning Research, vol. 5, pp. 14571469, 2004.

[16] D. L. Donoho, "For most large underdetermined systems of equations, the minimal \&1scr; $; \operatorname{sub}_{i j}$ font size $={ }^{\prime}-1_{i}{ }_{i j} /$ font $_{i j} /$ sub $_{i}$-norm near-solution approximates the sparsest near-solution," Communications on Pure and Applied Mathematics, vol. 59, no. 7, pp. 907-934, 2006.

[17] H. Cecotti and A. Gräser, "Time delay neural network with Fourier Transform for multiple channel detection of steady-state visual evoked potential for brain-computer interfaces," in Proc. European Signal Processing Conference (EUSIPCO), 2008.

[18] D. Krusienski, E. Sellers, D. McFarland, T. Vaughan, and J. Wolpaw, "Toward enhanced P300 speller performance," Journal of Neuroscience Methods, vol. 167, no. 1, pp. 15-21, Jan. 2008. 